\title{
HERRAMIENTAS DE ENTRENAMIENTO Y MONITORIZACIÓN PARA EL DESMINADO HUMANITARIO
}

\author{
Héctor Montes ${ }^{1,2}$, Roemi Fernández ${ }^{1}$, Pablo González de Santos ${ }^{1}$, Manuel Armada ${ }^{1}$ \\ ${ }^{1}$ Centro de Automática y Robótica CAR (CSIC-UPM); ${ }^{2}$ Facultad de Ingeniería Eléctrica, Universidad \\ Tecnológica de Panamá, \{hector.montes, roemi.fernandez, pablo.gonzalez, manuel.armada\}@csic.es
}

\begin{abstract}
Resumen
En este trabajo se presentan algunas herramientas para la monitorización y el entrenamiento en tareas de desminado humanitario, que han sido diseñadas $e$ implementadas en el Grupo de Robótica de Exteriores y de Servicios del Centro de Automática y Robótica dentro del marco del proyecto Europeo del 7PM “TIRAMISU” (Toolbox Implementation for Removal of Anti-personnel Mines, Submunitions and UXO). Básicamente, se han diseñado $e$ implementado cuatro herramientas para la monitorización, entrenamiento y localización de minas antipersonas simuladas e inertes debido a las competencias del grupo de trabajo. La primera de ellas es para la formación en el uso de detectores de metales, en donde se ha instrumentado para la monitorización en la realización de tareas especificas. La segunda, es dedicada a los e-tutores en los cuales se entrena al personal novato en los conceptos generales a gran escala del desminado humanitario y a la identificación de minas antipersonas y las primeras teoría para su desactivación. Otra herramienta diseñada $e$ instrumentada es la lanceta de búsqueda de minas enterradas (prodder), la cual tiene dos versiones, la última de ellas inalámbrica. Finalmente, la última herramienta es doble, la cual consta de un robot hexápodo semi-autónomo y tele-operado, que porta un brazo robótico instrumentado con sensores para explorar el terreno durante la localización de minas antipersonas enterradas.
\end{abstract}

Palabras Clave: Desminado humanitario, detector de metales, e-tutores, prodder, robot hexápodo, brazo explorador, instrumentación, herramientas de entrenamiento.

\section{INTRODUCCIÓN}

Según el Centro Internacional de Desminado Humanitario de Ginebra (GICHD) (https://www.gichd.org/), en 2004, en 15 programas de países diferentes afectados con campos de minas antipersonas, $292 \mathrm{~km}^{2}$ habían sido físicamente despejados, representando el $2.5 \%$ del área total contaminada por minas antipersonas enterradas. Esta estadística pone de manifiesto la ineficiencia en la lucha contra las minas antipersonas, en cuanto a la limpieza de éstas de los campos infestados, los cuales siguen siendo enormes [1]. Por otro lado, desde un punto de vista focalizado, Sri Lanka anunció la intención de adherirse a la Convención sobre la Prohibición de Minas Antipersonas (APMBC), después de una guerra civil de 26 años que ha dejado $64 \mathrm{~km}^{2}$ de tierra contaminada con minas, considerando que en 2020 el país estará libre de minas [2]. Esto indica que queda mucho por hacer en cuanto a estrategia y al uso de la tecnología en todos los sectores, ya que el proceso de desminado desde el mapeo de la tierra en busca de áreas sospechosas hasta el proceso de retirada de la mina es bastante largo.

Las situaciones en cada país son diferentes debido a las fuentes del conflicto, a las características climatológicas del mismo, al tipo de armamento utilizado, etc., por lo tanto, es complicado proporcionar soluciones estándares para todos los tipos de situaciones. En este caso particular, dentro del marco del proyecto TIRAMISU (http://www.fp7tiramisu.eu/) se han proporcionado ciertas herramientas y estudios de importancia para la acción en contra de las minas antipersonas.

En general, el proyecto TIRAMISU se dividió en diez módulos diferentes con la finalidad de abordar los objetivos propuestos en su plan de trabajo. No obstante, las herramientas de entrenamiento y de monitorización diseñadas y elaboradas por el Grupo de Robótica de Exteriores y de Servicios del CARCSIC-UPM, fueron desarrolladas en el módulo de "Detección cercana con base en el terreno" y en el de "Entrenamiento de usuarios finales, Centro de Acción Contra las Minas (MAC), Comunidad de I+D y personal clave".

Este escrito ha sido dividido en seis secciones, en la primera de ella se presenta, de manera muy breve, una introducción del trabajo presentado, considerando alguna información desarrollada por los estudios del GICHD, centro internacional de mayor importancia en la acción contra las minas, y el título de los módulos del proyecto TIRAMISU en donde se 
desarrollaron las herramientas descritas en este trabajo. En la sección 2 se presenta una herramienta de entrenamiento en el uso con detectores de metales, la cual fue instrumentada con unidades de medida inercial. Los tutores electrónicos con base a Adobe eLearning Suite se presentan en la sección 3, los cuales fueron validados por expertos en el área. En la sección 4 se describe la herramienta "prodder" que es una lanceta utilizada para detectar las minas que están enterradas, luego de haber sido localizadas por el detector de metales. El sistema robótico semiautónomo y tele-operado se describe, brevemente, en la sección 5, el cual consta de un robot hexápodo y un brazo de exploración. Finalmente, en la sección 6, se presentan algunas conclusiones.

\section{HERRAMIENTA DE ENTRENAMIENTO EN EL USO DE DETECTOR DE METALES}

La primera herramienta diseñada e implementada dentro del marco del proyecto TIRAMISU, por parte del Grupo de Robótica de Exteriores y de Servicio, fue la herramienta para monitorizar el uso de detectores de metales portátiles, con la finalidad de registrar el entrenamiento por parte de los aprendices en trabajos de localización de minas enterradas.

Esta herramienta de formación consta de dos partes principales, la primera de ellas es un sistema de seguimiento sensorial instalado en el detector de metales portátil, y la segunda es una interfaz gráfica de usuario (GUI), en donde se monitoriza y se registra la actividad de cada aprendiz en el uso de esta herramienta [3-4].

Para el diseño de esta herramienta se realizó un estudio de las variables que se deben tener en cuenta para la realización de búsqueda de minas antipersonas enterradas utilizando un detector de metales y con la entrevistas de expertos que formaban parte del consorcio de TIRAMISU. De esta manera, se seleccionaron las siguientes variables, consideradas de rendimiento crítico del instrumento: (i) la velocidad de escaneado, (ii) la distancia entre la cabeza del sensor del detector de metales y el suelo, (iii) la inclinación de la cabeza del sensor, (iv) paso de avance, $(v)$ tiempo de ejecución. Estas variables forman una base de datos que es una parte importante de la herramienta de capacitación mencionada, y además para evaluar el desempeño de los alumnos.

En la Figura 1 se presenta un detector de metales compacto VMC1 de la marca Vallon [5], el cual está instrumentado con una IMU y un seguidor de movimiento que tiene GPS incorporado, y un operador que tiene en sus pies dos IMUs con la finalidad de cuantificar el desplazamiento longitudinal de él cuando se realiza la tarea de escanear el terreno con el detector de metales.

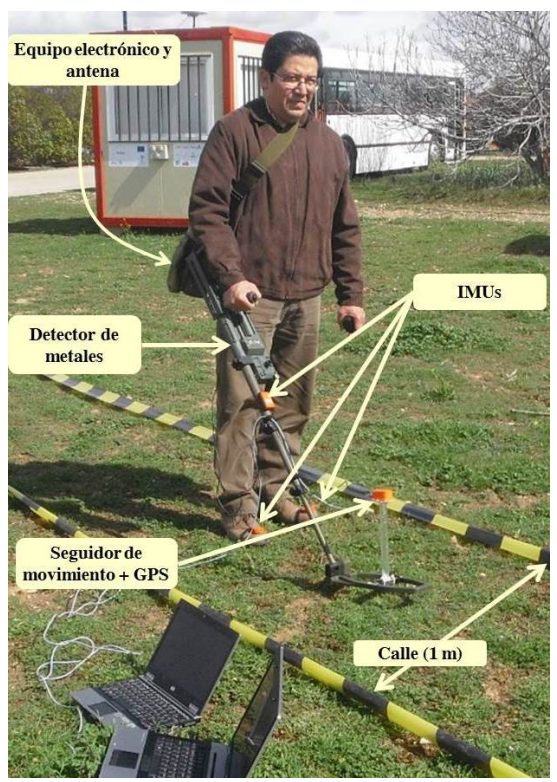

Figura 1. Aprendiz con la herramienta de entrenamiento para detectar minas antipersonas.

En la Figura 2 se presenta la interfaz gráfica de usuario (GUI) realizada en Matlab ${ }^{\circledR}$, la cual monitoriza las tareas llevadas a cabo por el aprendiz según las variables descritas anteriormente. Esta GUI se divide en seis partes principales, las cuales son: $(i)$ tipo de sesión, (ii) inicialización, (iii) bloque de control del proceso, (iv) ventana de monitorización del barrido, ( $v)$ monitorización de la velocidad, altura e inclinación del sensor, y (vi) bloque de exportación de los datos registrados.

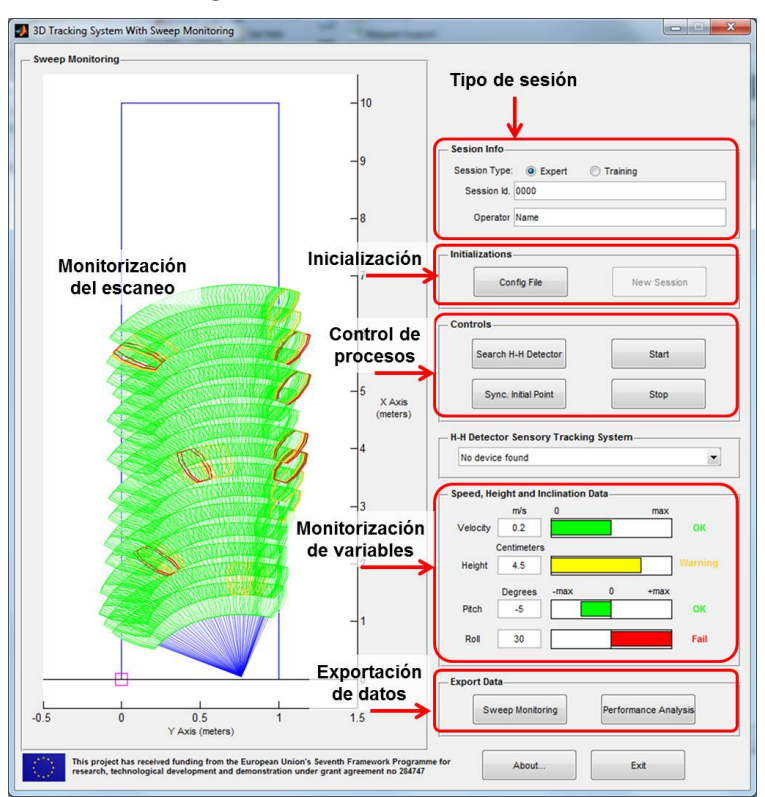

Figura 2. GUI para monitorizar las prácticas de uso del detector de metales. 


\section{HERRAMIENTA DE FORMACIÓN BASADA EN e- TUTORES}

Desde la última década y, más aún, en el último lustro, los procesos de enseñanza, formación o entrenamiento también hacen uso de la Tecnología de la Información y la Comunicación (TIC) para proporcionar el aprendizaje en una gran gama de temas y de centros de estudios [6-7]. Este tipo de educación está dentro del concepto de e-learning, que no trata solamente de tomar un curso y colocarlo en un ordenador, se trata de una combinación de recursos, interactividad, apoyo y actividades de aprendizaje estructuradas. Para realizar todo este proceso es necesario conocer las posibilidades y limitaciones que el soporte informático o plataforma virtual ofrece [8]. En consecuencia, se diseñó e implementó una herramienta, basada en e-tutores (concepto dentro del e-learning), con la finalidad de enseñar conceptos importantes en el reconocimiento de minas antipersonas, su ensamblaje, su desarme y neutralización, así como otros conceptos de estudios generales y no-técnicos para la localización de áreas sospechosas de minas antipersonas.

El primer e-tutor diseñado en el CAR y validado por grupos de expertos del proyecto TIRAMISU se titula "Estudio General Avanzado y Estudio No-Técnica", el cual está compuesto por varias secciones con la finalidad de instruir al aprendiz en los Centros de Acción Contra las Minas, en las primeras tareas relacionadas con todo el largo proceso de desminado humanitario. En la Figura 3 se presenta la página principal de este e-tutor desarrollado sobre Adobe eLearning Suite.

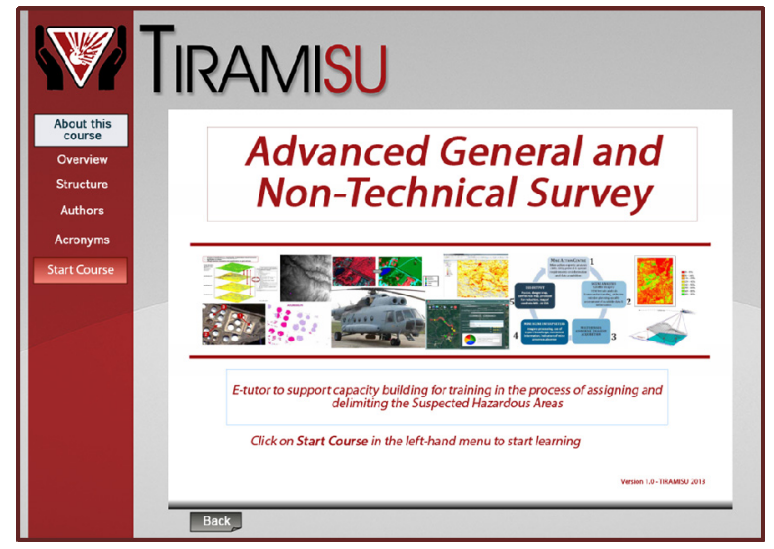

Figura 3. Portada del e-tutor "Advanced General and Non-Technical Survey".

Este e-tutor está compuesto por ocho lecciones independientes adecuadas para el aprendizaje asincrónico de cada estudiante. Cada lección es una secuencia de pantallas que incluyen texto, gráficos, videos e interactividad en forma de preguntas y comentarios. En cada lección se pretende alcanzar los objetivos de aprendizaje propuestos, utilizando instrucciones interactivas, paso a paso, y ejercicios que ayudan a reforzar lo que se pretende enseñar.

El segundo e-tutor ha sido desarrollado para ser utilizado en los MAC de los países que lo necesitan. La idea es proporcionar una formación inicial a las personas civiles que necesitan aprender acerca de la identificación de las minas terrestres antipersonas, y que requieren prepararse para trabajar en esta actividad [9]. Como es conocido, la formación por parte de los estudiantes y coordinada por los MAC, se puede realizar en cualquier momento $\mathrm{y}$ en cualquier lugar, ya que la base de la información es a través del aprendizaje electrónico. Este tutor electrónico se compone de sub-e-tutores, los cuales son: (i) Descripción general de las minas antipersonas, (ii) Descripción de los métodos comunes de remoción de minas, (iii) Descripción de la mina PMA-1, (iv) Descripción de la mina Valmara 69, (v) Descripción de la mina VS-50, y (vi) Descripción de la mina PPM-2.En la Figura 4 se presenta la portada de dos de los sub-e-tutores.

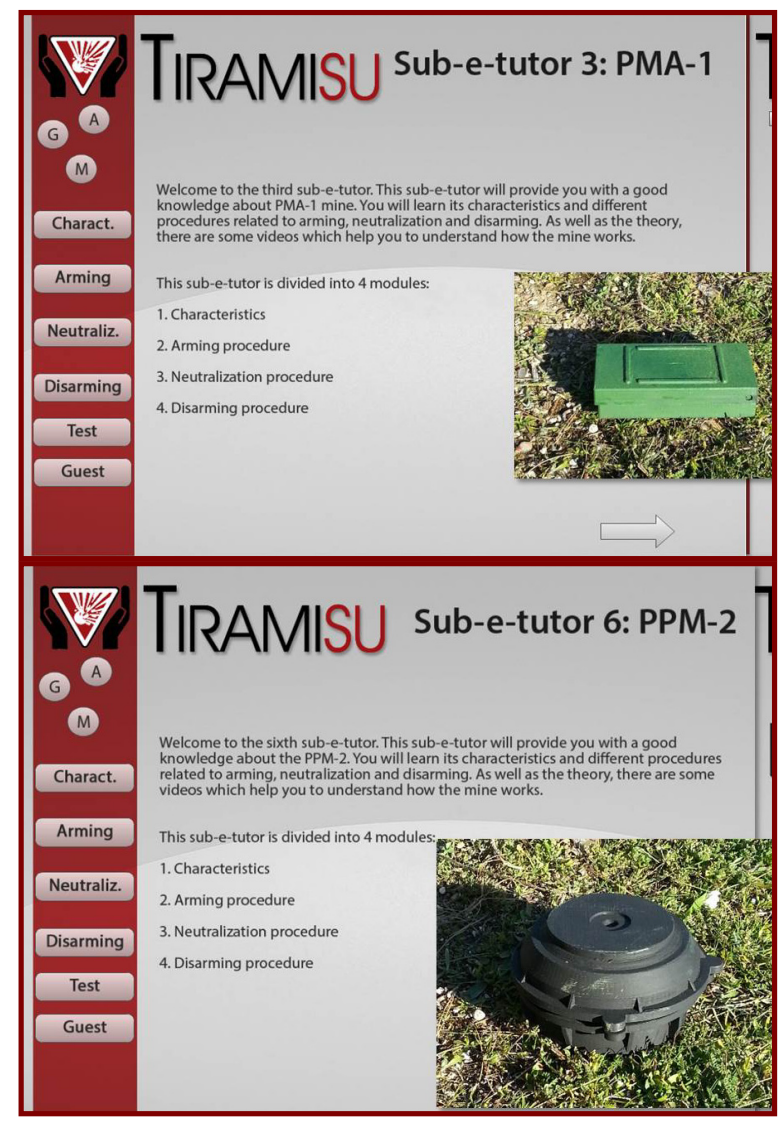

Figura 4. Portada de los sub-e-tutores 3 y 4, correspondientes al estudio de las minas PMA-1 y PPM-2, respectivamente. 


\section{HERRAMIENTA DE ENTRENAMIENTO PARA LA BÚSQUEDA DE MINAS ENTERRADAS}

Otra herramienta que ha sido diseñada dentro del marco del proyecto TIRAMISU es la de una lanceta instrumentada para la búsqueda directa de minas antipersonas enterradas, luego de ser localizada por el detector de metales u otro medio. Este dispositivo es conocido internacionalmente con su nombre en inglés "prodder", por lo tanto, es el que se utilizará de aquí en adelante. Se ha adquirido un prodder comercial de la casa Vallon [5], cuya parte principal es un cilindro de acero inoxidable afilado de unos $300 \mathrm{~mm}$ de longitud y de $8 \mathrm{~mm}$ de diámetro, que posteriormente, ha sido instrumentado con un sensor de fuerza y una unidad de medida inercial.

El objetivo de esta herramienta de formación es la de proporcionar datos de la fuerza ejercida por el operario cuando el prodder es insertado en el suelo y con qué inclinación con respecto al plano horizontal o paralelo al suelo en donde se encuentra enterrada la mina. Como es una herramienta de entrenamiento, el operario normalmente es un estudiante en fase de entrenamiento, o, previamente, un experto para obtener información útil de cómo se debe utilizar este tipo de herramienta, lo cual redundará en el aprendizaje de los novatos.

Algunas alarmas visuales (subsecuentemente, alarmas sonoras) son establecidas cuando las variables de fuerza e inclinación alcanzan ciertos límites predeterminados durante el proceso de entrenamiento. Estos límites son dependientes del tipo de mina que se está localizando y del tipo de terreno. En [10] se describe el diseño e implementación del primer prototipo de este instrumento de capacitación, además de algunos resultados experimentales.

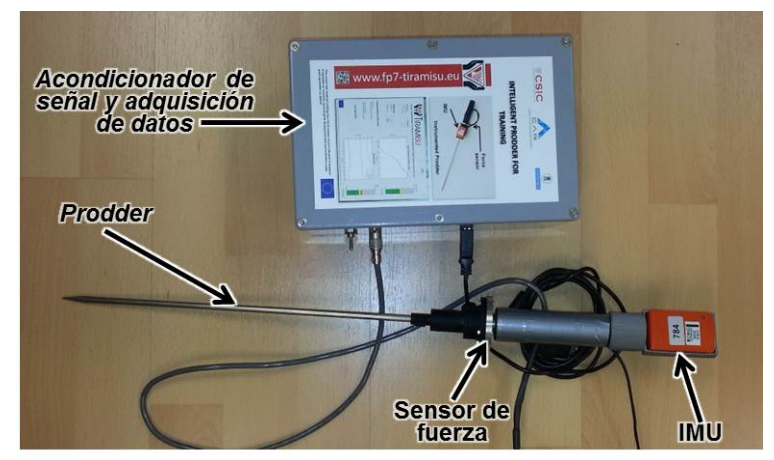

Figura 5. Vista del prodder instrumentado con la electrónica de soporte.
En la Figura 5 se presenta el primer prototipo de esta herramienta diseñada por el Grupo de Robótica de Exteriores y de Servicios. La IMU es utilizada para medir los ángulos de cabeceo, alabeo y guiñada durante el ejercicio de inserción del prodder en el suelo, al mismo tiempo que se mide la fuerza se inserción/presión de este dispositivo a lo largo de su eje.

Una segunda versión de esta herramienta puede ser revisada en [11], cuya modificación y mejora está relacionado con la portabilidad, esto es, que no depende de un ordenador cerca para poder transferir los datos desde la electrónica de soporte, que sí debe ser realizada en el prototipo mostrado en la Figura 5. La nueva herramienta es completamente inalámbrica y envía los datos a un sistema de adquisición de datos que está cerca del ordenador en la estación base. Se tiene una ventaja, que es que el supervisor de la clase podría observar a varios aprendices realizando sus entrenamientos en diferentes lugares del campo de trabajo sin que ninguna conectividad cableada pueda interferir entre ellos y con un solo ordenador, el del supervisor. La interfaz gráfica de usuario sería la misma para ambos prototipos y es mostrada en la Figura 6.

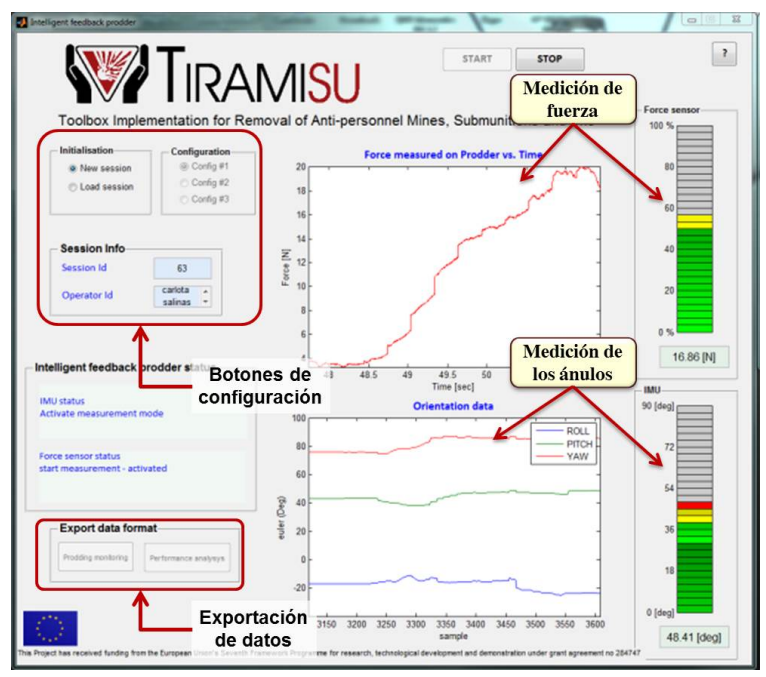

Figura 6. Interfaz para monitorizar las prácticas de los estudiantes con el uso del prodder.

\section{DESCRIPCIÓN DEL SISTEMA ROBÓTICO PARA LA BÚSQUEDA Y DETECCIÓN DE MINAS ANTIPERSONAS}

El sistema robótico para la búsqueda y detección de minas antipersonas diseñado, desarrollado y modificado por el Grupo de Robótica de Exteriores y de Servicios consiste de un robot caminante de seis patas y de un brazo de exploración de cinco grados 
de libertad, el cual porta en su extremo un detector de metales. Se ha utilizado, para este sistemaherramienta, un robot con patas debido a que es un sistema prometedor para la búsqueda de minas antipersonas en comparación con robots con ruedas, porque puede sortear los obstáculos una vez encontrados (posibles minas en su paso) y seguir con la búsqueda. Existen algunos prototipos de robots con patas que han sido probados experimentalmente, los cuales pueden ser vistos en [12-16].

El robot hexápodo diseñado en el CAR tiene una arquitectura de control que consiste en un ordenador a bordo, tarjetas de control, tarjetas de potencia, sistema de adquisición de datos, tarjetas de acondicionamiento de señal, sensores de posicionamiento, motores de CC, Wi-Fi, DGPS, y otros dispositivos y accesorios. Los algoritmos diseñados han sido desarrollados en $\mathrm{C} / \mathrm{C}++\mathrm{y}$ ejecutados en tiempo real por el sistema operativo QNX. Con ello se han realizado varias estrategias de control para llevar a cabo tareas de modos de caminar en paralelo con las actividades de escaneo del brazo de exploración para la búsqueda y localización de minas antipersonas, considerando las variables señaladas en la sección 2 [17].

El objetivo es que el robot realice trayectorias estables con el fin de que el brazo de exploración pueda realizar movimientos adecuados de su efector final, donde está montado el cabeza del detector de metales. Para este fin, el robot hexápodo ejecuta pasos pre-establecidos hacia una posición fija de su centro de masa, para que el brazo manipulador realice la exploración del suelo. En la Figura 7 se muestra el robot hexápodo con el brazo de exploración instalado en la parte frontal de su cuerpo.

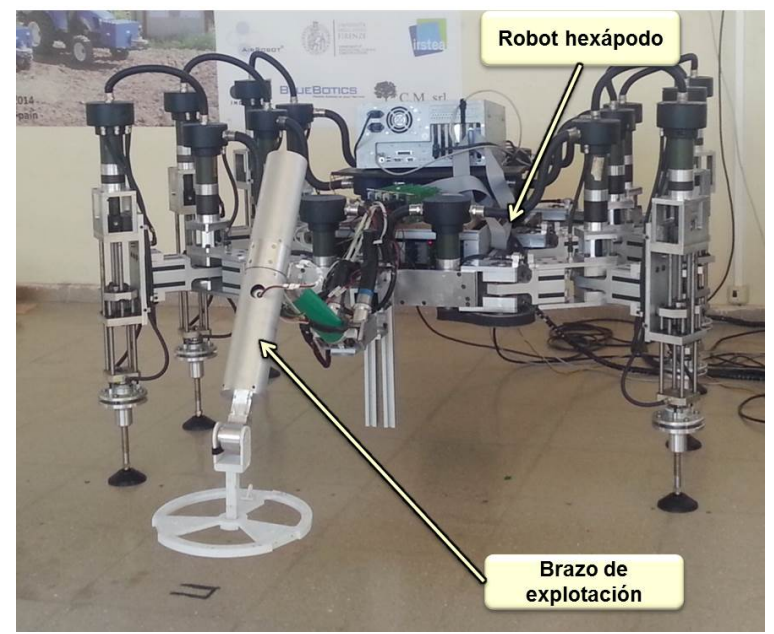

Figura 7. Sistema robótico para búsqueda de minas antipersonas, compuesto por un robot hexápodo y un brazo de exploración.
El brazo manipulador de exploración lleva instalado dos tipos de sensores diferentes, una mini cámara de tiempo de vuelo (ToF) y un sistema multisensorial VRMagic. La primera cámara tiene una resolución de $120 \times 160$ píxeles utilizada para obtener un mapa de profundidad, una imagen de amplitud y una nube de puntos que contienen las coordenadas cartesianas del objetivo que se está explorando.

La segunda cámara está equipada con dos sensores CMOS síncronos, que adquieren imágenes RGB con una resolución de 754 × 480 píxeles, cada uno. La fusión sensorial entre los datos de la mini-ToF con las imágenes RGB proporciona la información necesaria para conocer el área de exploración que realiza el brazo manipulador. La Figura 8 muestra algunas imágenes/datos obtenidos por el sistema sensorial instalado en el brazo manipulador.

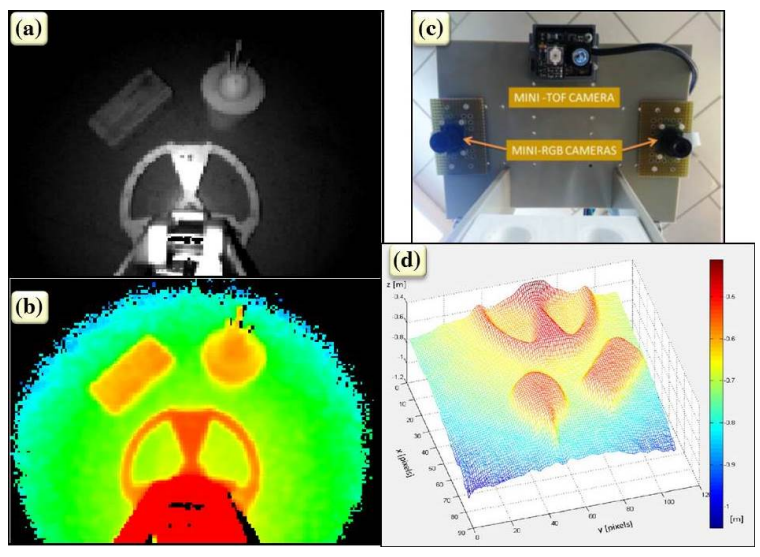

Figura 8. Sistema sensorial instalado en el brazo de exploración. (a) Imagen de amplitud de la mini ToF, (b) datos de rango de profundidad de la mini ToF, (c) sistema sensorial compuesto por una mini-ToF y dos sensores mini-RGB, (d) mapeo del área escaneada.

\section{CONCLUSIÓN}

Este artículo ha presentado un resumen de las tareas principales que han sido desarrolladas dentro del marco del proyecto TIRAMISU, por parte del Grupo de Robótica de Exteriores y de Servicios del CAR CSIC-UPM, con la finalidad de presentar al lector una información de interés actual, la cual puede ampliar leyendo las referencias citadas y consultado a los autores de este trabajo. Los trabajos realizados se han centrado en el diseño, implementación y validación de varias herramientas que han sido propuestas para ser utilizadas en las fases requeridas del desminado humanitario. Las herramientas desarrolladas poseen un nivel de TRL del 5 y 6, lo cual ha estado muy bien dentro de los requerimientos del proyecto. 


\section{Agradecimientos}

Este trabajo ha sido financiado por el proyecto TIRAMISU con Convenio de Subvención No 284747 del 7PM de la UE, y parcialmente financiado por el proyecto RoboCity2030-III-CM (Robótica aplicada a la mejora de la calidad de vida de los ciudadanos Fase III, S2013 / MIT-2748), financiado por Programas de Actividades I+D de la Comunidad de Madrid y cofinanciado por los Fondos Estructurales de la UE; y por el proyecto del CSIC Robótica y Sensores para los Retos Sociales (ROBSEN) (PIE 20165E050).

\section{Referencias}

[1] GICHD, (2009) Annual Report 2009. Geneva International Centre for Humanitarian Demining. Geneva, Switzerland.

[2] GICHD, (2015) Annual Report 2015. Geneva International Centre for Humanitarian Demining. Geneva, Switzerland.

[3] Fernández, R., Montes, H., Salinas, C., González de Santos, P., Armada, M., (2012) Design of a training tool for improving the use of hand-held detectors in humanitarian demining. Industrial Robot: An International Journal, 39(5): 450 463.

[4] Fernández, R., Montes, H., Gusano, J., Sarria, J., Armada, M., (2014) Design of the Human Machine Interface for training activities with hand-held detectors. In Proc. International Symposium "Mine Action 2014", 23-25 April 2014, Zadar, Croatia, pp. 115-120.

[5] Vallon, (2012) VMC1 Mine Detector. Disponible en: http://www.vallon.de/ pdf/VMC1_leaflet _10_2012.pdf.

[6] Mellar, H., Kambouri, M., Logan, K., Betts, S., Nance, B. and Moriarty, V., (2007) Effective Teaching and Learning: Using ICT. London: NRDC.

[7] Wu, J. P., Tsai, R. J., Chen, C. C., and Wu, Y. C., (2006) An integrative model to predict the continuance use of electronic learning systems: hints for teaching. International Journal on ELearning, 5(2), 287-302.

[8] Belloch, C., (2012) Entornos Virtuales de Aprendizaje. Obtenido de Recursos Tecnológicos en Educación y Logopedia: http://www.uv.es/bellochc/pedagogia/EVA3.pdf

[9] Montes, H., Díaz, E., Fernández, R., Sarria, J., Armada, M., (2015) e-Tutor for training in antipersonnel landmines identification. In Proc. 12th Intl. Symposium MINE ACTION 2015, 2730 April, Biograd, Croatia, pp 189-192.

[10]Fernández, R., Montes, H., Gusano, J., Sarria, J., and Armada, M., (2014) Force and angle feedback prodder. In Proc. 17th Intl. Conf. on Climbing and Walking Robots and the Support Technologies for Mobile Machines. July 21-23, Poznan, Poland. Mobile Service Robotics, pp. 305-312.

[11] Montes, H., Fernández, R., De Lorenzo, D., and Armada, M., (2016) Design and implementation of a wireless prodder for instructional purposes in landmine detection. In Proc. 19th Intl. Conf. on Climbing and Walking Robots and the Support Technologies for Mobile Machines. Sept. 12-14, London, UK. Advances in Cooperative Robotics: pp. 348-355.

[12] Gonzalez de Santos, P. and Jimenez, M.A., (1995) Generation of discontinuous gaits for quadruped walking machines. Journal of Robotics Systems, 12(9): 599-611.

[13] Habumuremyi, J.C., (1998) Rational designing of an electropneumatic robot for mine detection. In Proc. Of the $1^{\text {st }}$ Intl. Conference on Climbing and Walking Robots CLAWAR'98, Brussels, Belgium, November 26-28, pp. 267-273.

[14]Hirose, S. and Kato, K., (1998) Quadruped walking robot to perform mine detection and removal task. In Proc. of the 1st International Conference on Climbing and Walking Robots, Brussels, Belgium, pp. 261-266.

[15] Nonami, K., Huang, Q., Komizo, D., Fukao, Y., Asai, Y., Shiraishi, Y., Fujimoto, M., Ikedo, Y., (2003) Development and control of mine detection robot Comet-II and Comet-III. JSME International Journal, Series C, 46(3): 881-890.

[16]Gonzalez de Santos P, Cobano J, Garcia E, Estremera J, Armada M., (2007) A six-legged robot-based system for humanitarian demining missions. Mechatronics, 17: 417-430.

[17] Montes, H., Mena, L., Fernandez, R., Sarria, J., and Armada, M., (2015) Inspection platform for applications in humanitarian demining. In Proc. 18th Intl. Conf. on Climbing and Walking Robots and the Support Technologies for Mobile Machines. Sept. 6-9, HangZhou, China. Assistive Robotics, pp. 446-453. 